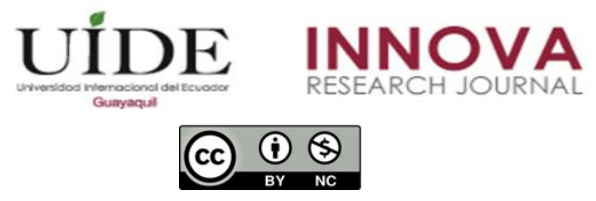

INNOVA Research Journal, ISSN 2477-9024

(Septiembre-Diciembre, 2019). Vol. 4, No.3 pp.158-169

DOI: https://doi.org/10.33890/innova.v4.n3.2019.944

URL: http://revistas.uide.edu.ec/index.php/innova/index

Correo: innova@uide.edu.ec

\title{
Arsénico en aguas, suelos y sedimentos de la Reserva Biológica de Limoncocha - Ecuador con fines de conservación
}

\section{Arsenic in waters, soils and sediments of the Limoncocha Biological Reserve - Ecuador for conservation purposes}

\author{
Katty Verónica Coral Carrillo \\ Universidad Internacional SEK, Ecuador \\ Universidad de Cantabria, España \\ Dayana Carrillo \\ Miguel Martínez Fressneda \\ Universidad Internacional SEK, Ecuador
}

Jorge Esteban Oviedo

Universidad de Cantabria, España

Autor para correspondencia: katty.coral@uisek.edu.ec; jorjandrito@hotmail.com

Fecha de recepción: 18 de diciembre de 2018 - Fecha de aceptación: 28 de mayo de 2019

Resumen: La Reserva Biológica de Limoncocha (RBL) en Ecuador, es de gran importancia ecológica, alberga especies de flora y fauna de importante interés científico. Por su ubicación geográfica, presenta riesgo de contaminación de sus componentes ambientales: agua, suelo y sedimentos, debido a la explotación petrolera, la agricultura y otras actividades antropogénicas características de la zona. La presencia de varios campos petroleros productivos así como plantaciones agrícolas en la zona, son de vital importancia para la economía ecuatoriana, sin embargo, producen vertidos sólidos, líquidos y gaseosos, con concentración de sustancias tóxicas, mismas que deben ser evaluadas y contrarrestadas, tal es el caso del Arsénico. El presente estudio evalúo la concentración de este elemento en los factores ambientales mencionados en la RBL, a través de su detección y cuantificación por análisis químico utilizando Absorción Atómica con generación de hidruros a partir de muestras recogidas entre abril de 2015 y abril de 2016. Los datos sobre las concentraciones de esta sustancia se compararon con la ley ecuatoriana y con valores referenciales internacionales para determinar si los habitantes de la RBL, en su mayoría de etnia kichwa, presentan exposición por las actividades que desarrollan en los factores ambientales estudiados. Permitiendo, establecer el inventario ambiental del área y disponer de valores referenciales para determinar el manejo más adecuado de esta reserva natural.

Palabras Claves: aguas; áreas naturales; arsénico; contaminación; sedimentos; suelo

\begin{abstract}
The Limoncocha Biological Reserve (RBL) in Ecuador is of considerable ecological importance, and it hosts species of flora and fauna of significant scientific interest. Due to its geographical location, it presents a risk of contamination of its environmental components: water, soil, and sediments, due to oil exploitation, agriculture, and other anthropogenic activities
\end{abstract}


characteristic of the area. The presence of several productive oil fields, as well as agricultural plantations in the area, are of vital importance for the Ecuadorian economy; however, they produce reliable, liquid and gaseous discharges, with a concentration of toxic substances, which must be evaluated and counteracted, such This is the case of Arsenic. The present study assessed the level of this element in the environmental factors mentioned in the RBL, through its detection and quantification by chemical analysis using Atomic Absorption with the generation of hydrides from samples collected between April 2015 and April 2016. Data on the concentrations of this substance were compared with the Ecuadorian law and with international reference values to determine if the inhabitants of the RBL, mostly of Kichwa ethnic group, present exposure for the activities they develop in the environmental factors studied. Allowing, establish the environmental inventory of the area and have reference values to determine the most appropriate management of this natural reserve.

Key Words: natural areas; landscape; ecosystems; pollution; waste; soil

\section{Introducción}

La Reserva Biológica de Limoncocha (en adelante, RBL) localizada en el cantón Shushufindi, Provincia de Sucumbíos, al nororiente de la Amazonía Ecuatoriana presenta gran importancia ecológica, pues alberga especies de flora y fauna de elevado interés científico, sin embargo, se encuentra sometida a un peligro potencial de contaminación de sus recursos agua, suelos y sedimentos debido a las actividades extractivas de petróleo, agrícolas y antropogénicas propias de la zona. La presencia de bloques petroleros con varios campos productivos, así como la actividad agrícola de la zona, son de vital importancia para la economía nacional, sin embargo, son actividades que generan impactos ambientales a nivel local y global, produciendo destrucción de la biodiversidad y del ambiente en general (Bravo, 2007). Según Neira (2006), el $81,8 \%$ de la población kichwa que vive en la RBL depende de la biodiversidad como medio de subsistencia (agricultura, cacería y pesca); estas actividades se desarrollan en las aguas, suelos y sedimentos de la RBL, por lo que su potencial contaminación puede afectar al medio de subsistencia de la comunidad, así como a su salud. El arsénico es un no metal, cuyas sales se encuentran en forma natural en la corteza terrestre; sin embargo cuando se liberan al ambiente por las actividades humanas, pueden llegar a convertirse en contaminantes del aire, agua superficial, sedimentos, suelo y otros ambientes naturales (Reyes et al, S.A).

El arsénico no puede ser destruido en el ambiente, solamente puede cambiar de forma. Muchos compuestos comunes de arsénico pueden disolverse en agua. La mayor parte del arsénico en el agua terminará eventualmente en el suelo o el sedimento (ATSDR, 2014), la presencia de este elemento no siempre es sinónimo de contaminación antropogénica; sin embargo su cuantificación es fundamental, pues si bien puede existir de forma natural, excesos en su concentración tienen efectos nocivos para la salud humana y ambiental.

La exposición a dosis elevadas de compuestos de arsénico inorgánico puede producir síntomas de conjuntivitis, bronquitis y disnea, seguidos por molestias gastrointestinales y vómitos, posteriormente síntomas cardíacos y shock irreversible. En un caso fatal se describen niveles de arsénico en sangre superior a $3 \mathrm{mg} / \mathrm{L}$ (Nordberg, 2014). 
En los casos de una exposición a largo plazo de arsénico a través de la comida o agua, los síntomas son diarrea o estreñimiento, enrojecimiento de la piel, pigmentación e hiperqueratosis, trastorno caracterizado por el engrosamiento de la capa externa de la piel, además de presentarse afectación vascular. En la Intoxicación crónica por arsénico es habitual la anemia, la leucopenia y daño hepático (Nordberg, 2014).

Según la Agencia Internacional para la Investigación sobre el cáncer (IARC) clasifica a los compuestos de arsénico inorgánico como cancerígenos pulmonares y cutáneos. Además de presentar una mayor incidencia de angiosarcoma hepático y cáncer de estómago, a dosis elevadas presenta efectos teratogénicos. Los arsénicos orgánicos pueden ser tóxicos, aunque sus efectos adversos no están totalmente documentados en humanos. Cantidades de arsénico orgánico presentes en pescados y mariscos pueden consumirse sin efectos nocivos, estos se excretan rápidamente, principalmente por la orina (Nordberg, 2014).

El arsénico se encuentra naturalmente en el suelo y en minerales y, por lo tanto, puede entrar al aire, al agua y a suelos en otras áreas en polvo que levanta el viento y puede entrar al agua en efluente de lluvia o en agua que se filtra a través del suelo (ATSDR, 2014).

La Universidad Internacional SEK cuenta con una estación en la RBL desde 1998, la cual ha servido como base para la realización de múltiples investigaciones tanto de carácter ambiental como social. Una de estas investigaciones es el estudio llevado a cabo en el año de 1998, en la que se realizó una caracterización y evaluación de suelos y sedimentos del sector de la laguna de Limoncocha, realizada por María Beatriz Cordovez, con el que se estableció el diagnóstico del estado de la RBL para la última década del siglo pasado, pero sin tomar en cuenta el Arsénico. Pese a todos los trabajos previos, no existen investigaciones actuales sobre la evaluación de metales pesados y no metales en aguas, suelos y sedimentos, por lo que el presente estudio pretende desarrollar una línea base de la potencial presencia del arsénico en aguas, suelos y sedimentos de la Reserva Biológica Limoncocha (RBL), herramienta que servirá para implementar medidas para la conservación de esta área protegida.

Los valores de fondo de arsénico en ríos y aguas superficiales, a nivel iberoamericano, son relativamente bajos, en general, inferiores a $0,8 \mu \mathrm{g} / \mathrm{L}$ aunque pueden variar dependiendo de factores como recarga (superficial y subterránea), litología de la cuenca, drenaje de zonas mineralizadas, clima, actividad minera y disposición de residuos urbanos y/o industriales. (Alarcón et al, 2013)

En el Ecuador, el acuerdo Ministerial 097 A establece los siguientes valores referenciales de As, en aguas y suelos, $0.05 \mathrm{mg} / \mathrm{L}$ y $12 \mathrm{mg} / \mathrm{kg}$ respectivamente, al no existir un valor referencial para sedimentos en el país, se tomó de referencia el valor de $5.9 \mathrm{mg} / \mathrm{kg}$, establecido en el ISQG. 2001. "Canadian Sediment Quality Guidelines for the Protection os aquatic life: Summary tables"

\section{Área de Estudio}

La RBL se encuentra a una altura promedio de 230 msnm y una extensión de 4613,25 ha, constituyendo una de las áreas del Sistema Nacional de Áreas Protegidas más pequeñas del país. 
La temperatura media anual del lugar es $25^{\circ} \mathrm{C}$, llegando a un máximo de $32^{\circ} \mathrm{C}$ y registrándose un mínimo de $16^{\circ} \mathrm{C}$, recibe una precipitación anual de 3065mm (Madera, 2011).

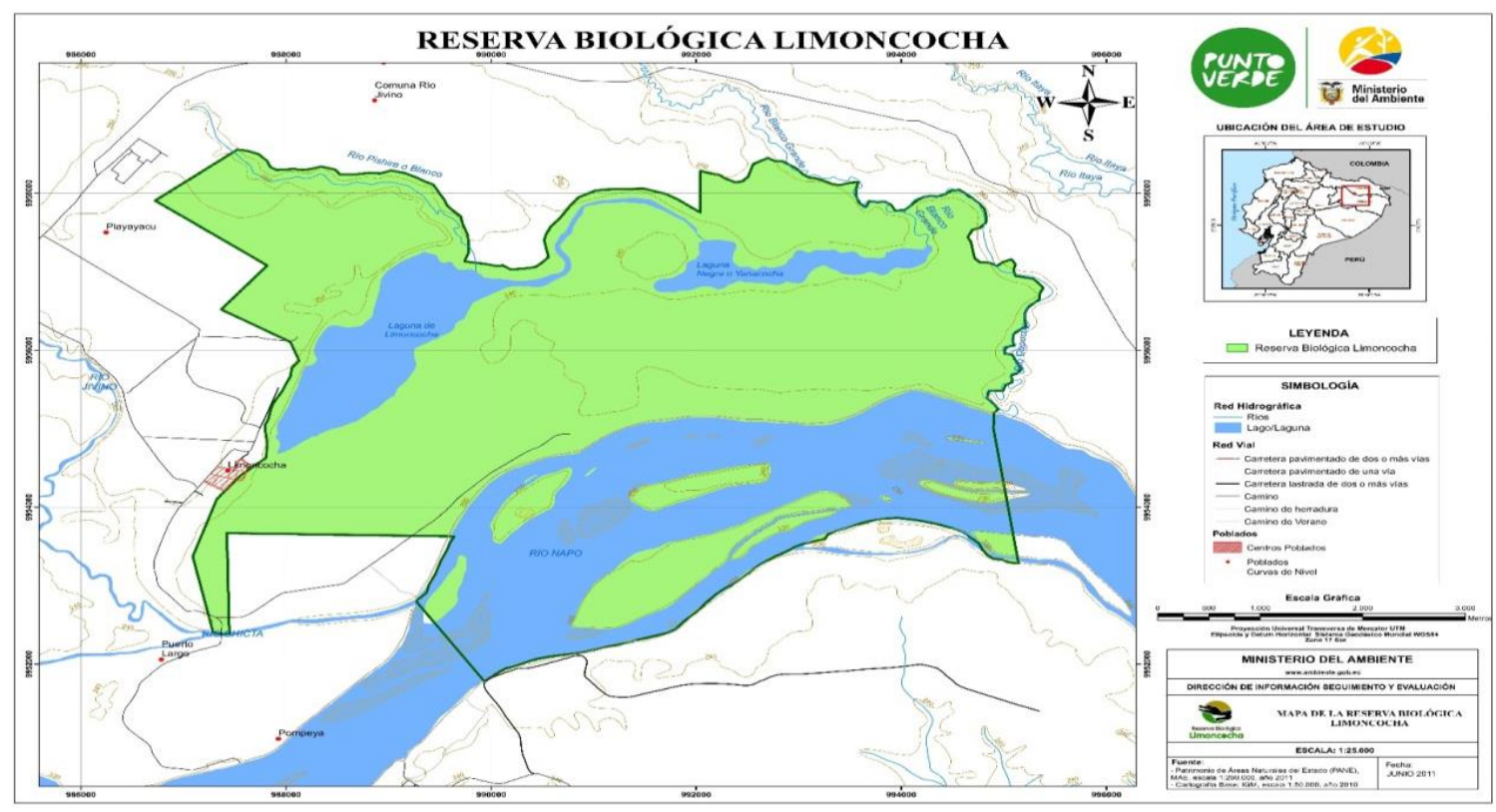

Figura 1. Ubicación Reserva Biológica Limoncocha (MAE, 2011)

La creación de la Reserva Biológica Limoncocha (RBL) se llevó a cabo en 1985, el mismo año en que el Estado Ecuatoriano expulsó a la misión evangélica norteamericana conocida como el Instituto Lingüístico de Verano (ILV), que desarrollaba en varios sectores de la Amazonía, procesos de evangelización y apoyo social para comunidades indígenas, iniciándose en este lugar los primeros acercamientos con el pueblo Waorani (Armas \& Lasso, 2011).

La Reserva Biológica de Limoncocha fue declarada como tal el 23 de Septiembre de 1985, mediante el acuerdo ministerial No. 394, publicado en el registro oficial No. 283 y declarada como sitio RAMSAR en 1998 (MAE, 2015), la asignación de la RBL como Humedal RAMSAR comprende una categorización de humedal continental “O”, que significa Lagos permanentes de agua dulce (de más de 8 ha) e incluye grandes madre viejas (meandros o brazos muertos de ríos) (Madera, 2011).

La RBL, de igual manera, forma parte del Área de Importancia para la Conservación de las Aves (IBA) No. EC039, reconocida oficialmente como tal, mediante el acuerdo ministerial No. 001 del primero de marzo de 2005. Desde la perspectiva de su condición de Reserva Biológica, sitio RAMSAR e IBA, se considera de fundamental importancia de conservación (Armas \& Lasso, 2011). 


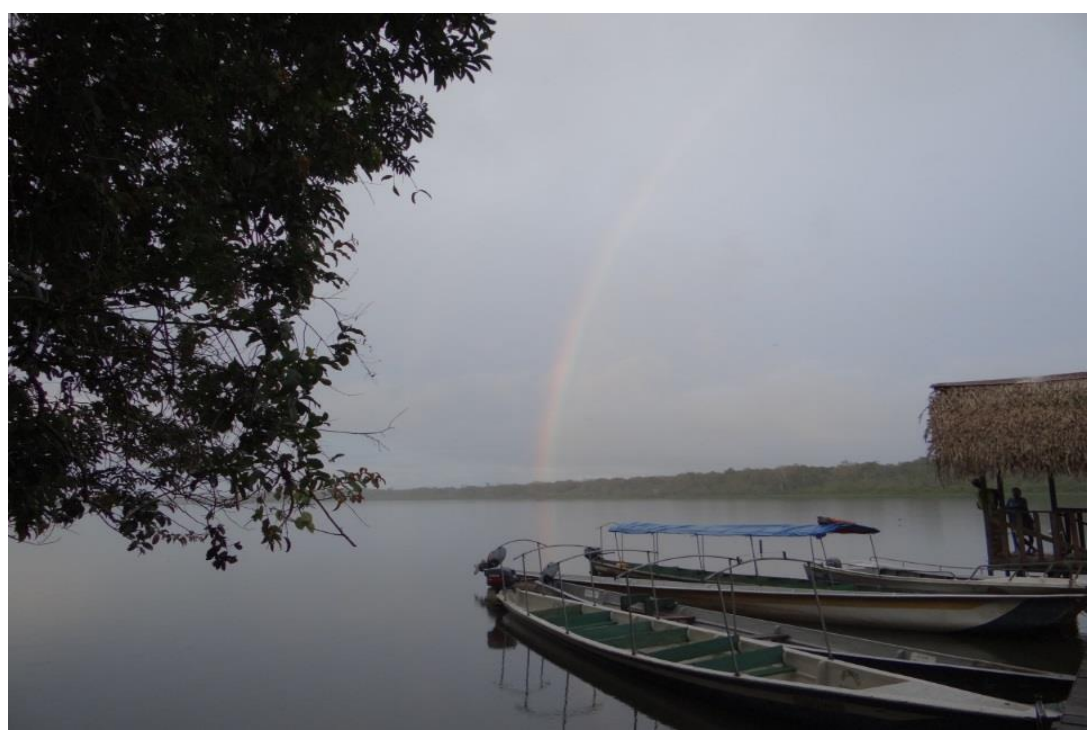

Figura 2. Laguna de Limoncocha (Fotografía K Coral)

\section{Materiales y métodos}

\section{Muestreo}

En una primera salida de reconocimiento se establecieron los puntos de muestreo de los tres factores ambientales agua, suelo y sedimentos, estos se escogieron con un criterio de mayor potencial de contaminación antropogénica, obteniendo así cinco estaciones (sitios) de la laguna de Limoncocha: Caño, Desembocadura Pishira, Desembocadura Playayacu, zona profunda de la laguna y Muelle. También se determinaron puntos de muestreo en el Río Napo (Blanco), río Pishira y río Playayacu, en zonas aledañas a la laguna.

El muestreo de agua superficial y sedimentos se inició en el mes de abril del 2015 al mes de abril del 2016, con un total de 10 salidas de muestreo, realizándose muestreos in situ de pH, conductividad eléctrica y temperatura. Para la recolección de las muestras de sedimento se utilizó un nucleador de sedimentos de acero inoxidable de $350 \mathrm{~mm}$ de longitud y un diámetro de 50 mm. Los sitios fueron geo posicionados y registrados en el mapa correspondiente. Los puntos escogidos para el muestreo de suelos se seleccionaron en base a la modalidad a "criterio: zonas antropogénicamente intervenidas" en función del reconocimiento inicial realizado, obteniendo así seis puntos, los cuales son: Pozo antiguo, Pozo Laguna, Pozo Jivino B, Instituto, la Estación Científica de la RBL y como blanco se escogió un punto en el sendero "El Caimán", debido a que se trata de una zona no intervenida por la actividad antropogénica, para la recolección de las muestras se utilizó un barreno de acero inoxidable y $40 \mathrm{~cm}$ de largo. 


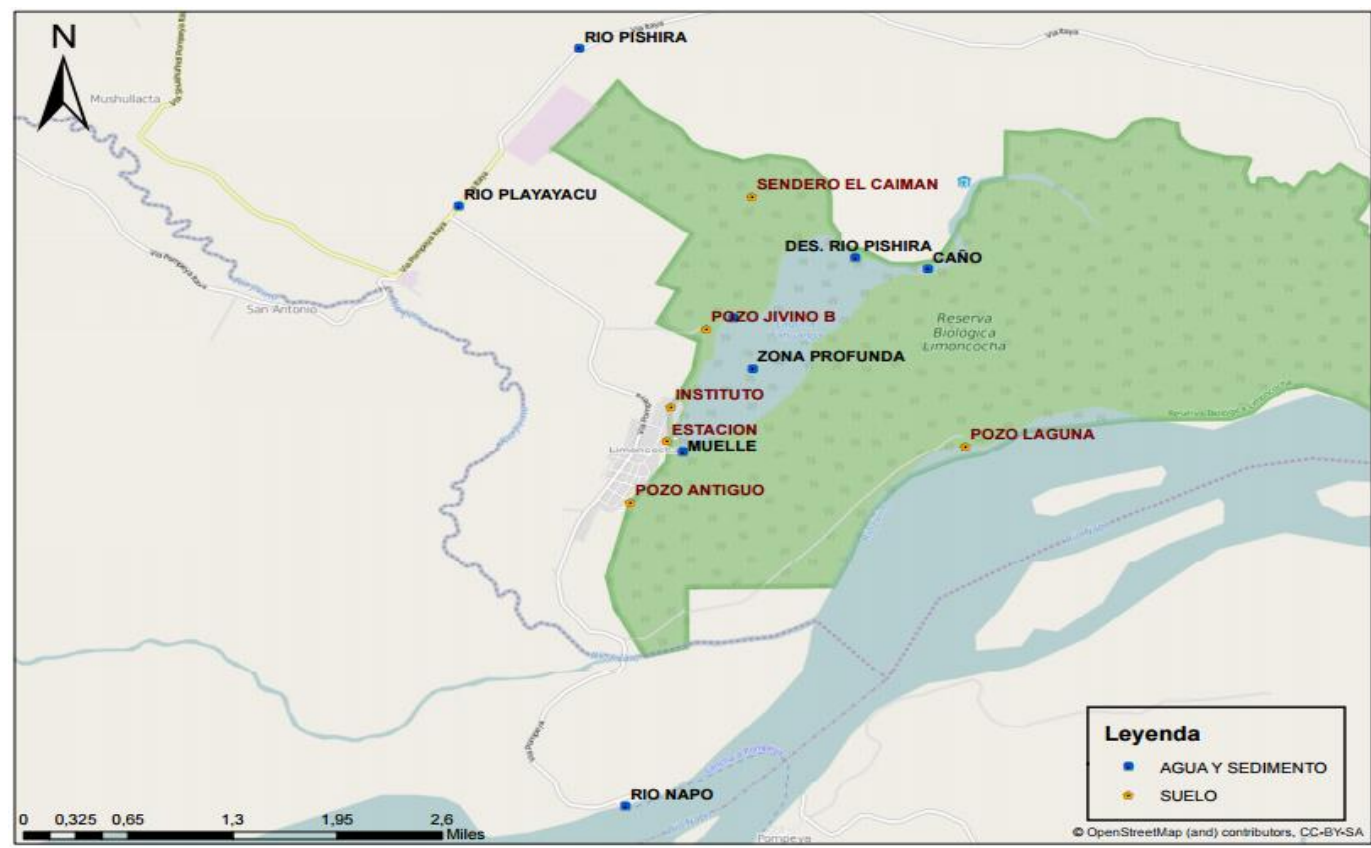

Figura 3. Mapa de Ubicación de los Puntos de muestreo para Aguas, Sedimentos y Suelos. (Elaborado por Carrillo \& Cerón, 2016)

\section{Determinación Analítica}

En primer lugar las muestras recibieron un pretratamiento para su conservación, acidificando las muestras de agua y secando los suelos y sedimentos, y una posterior pulverización. La digestión de aguas se utilizó el método: STANDARD METHOD 3030 E "Digestión de metales por ácido nítrico en aguas" (APHA AWWA WEF, Standard Methods, 2005). Para la determinación de metales pesados en suelos y sedimentos muestreados en la RBL, se seleccionó el método: EPA 3050B “Acid Digestion Of Sediments, Sludges, And Soils” (EPA, 1996).

Las muestras digeridas se llevaron a lectura de los metales deseados por absorción atómica con generador de hidruros, con un límite de detección de $1 \mu \mathrm{g} / \mathrm{L}$, para aguas y 0.001 $\mathrm{mg} / \mathrm{kg}$ para suelos y sedimentos.

\section{Fondo Geoquímico}

Para la realización de este estudio se tomó en cuenta los niveles de fondo, que son trazas de metales existentes en suelos y sedimentos de forma natural, sin embargo las actividades antropogénicas incrementan estos niveles de concentración de metales en suelos y sedimentos con respecto a los niveles de fondo (Hernández-Crespo et al, 2015).Como fondo geoquímico se obtuvo una muestra de sedimento a $50 \mathrm{~cm}$ de profundidad en la zona más profunda de la Laguna de Limoncocha, el valor obtenido de As en esta muestra fue comparado con las medianas de los puntos de sedimentos a fin de determinar si existe contaminación por As. Resultados y discusión. 
En todos los puntos de muestreo para aguas, los niveles registrados se encontraban por debajo del límite de detección del equipo de absorción atómica con generador de Hidruros, es decir, valores menores a $1 \mathrm{ppb}$ o $1 \mu \mathrm{g} / \mathrm{L}$.

Los datos de concentración en $\mathrm{mg} / \mathrm{kg}$ de arsénico en sedimentos de la RBL, fueron obtenidos mediante espectrofotometría de absorción atómica por generador de hidruros, técnica utilizada debido a su elevada sensibilidad para ultratrazas de metales como es el arsénico. Los datos obtenidos de concentración de As en sedimentos de la RBL tienen como valor mínimo $0,117 \mathrm{mg} / \mathrm{kg}$ en el punto río Playayacu y como valor máximo $1,960 \mathrm{mg} / \mathrm{kg}$ en el punto desembocadura Pishira, observando dos valores atípicos en el punto río Napo y en el punto río Playayacu; mientras que temporalmente, el valor máximo se encuentra el mes de diciembre y el mínimo en abril del 2015. De los datos de concentración de abril del 2015 a abril 2016, seis valores fueron no detectables por el método utilizado, los valores promedios de As en sedimentos varían entre 0.300 y $1.300 \mathrm{mg} / \mathrm{kg}$.

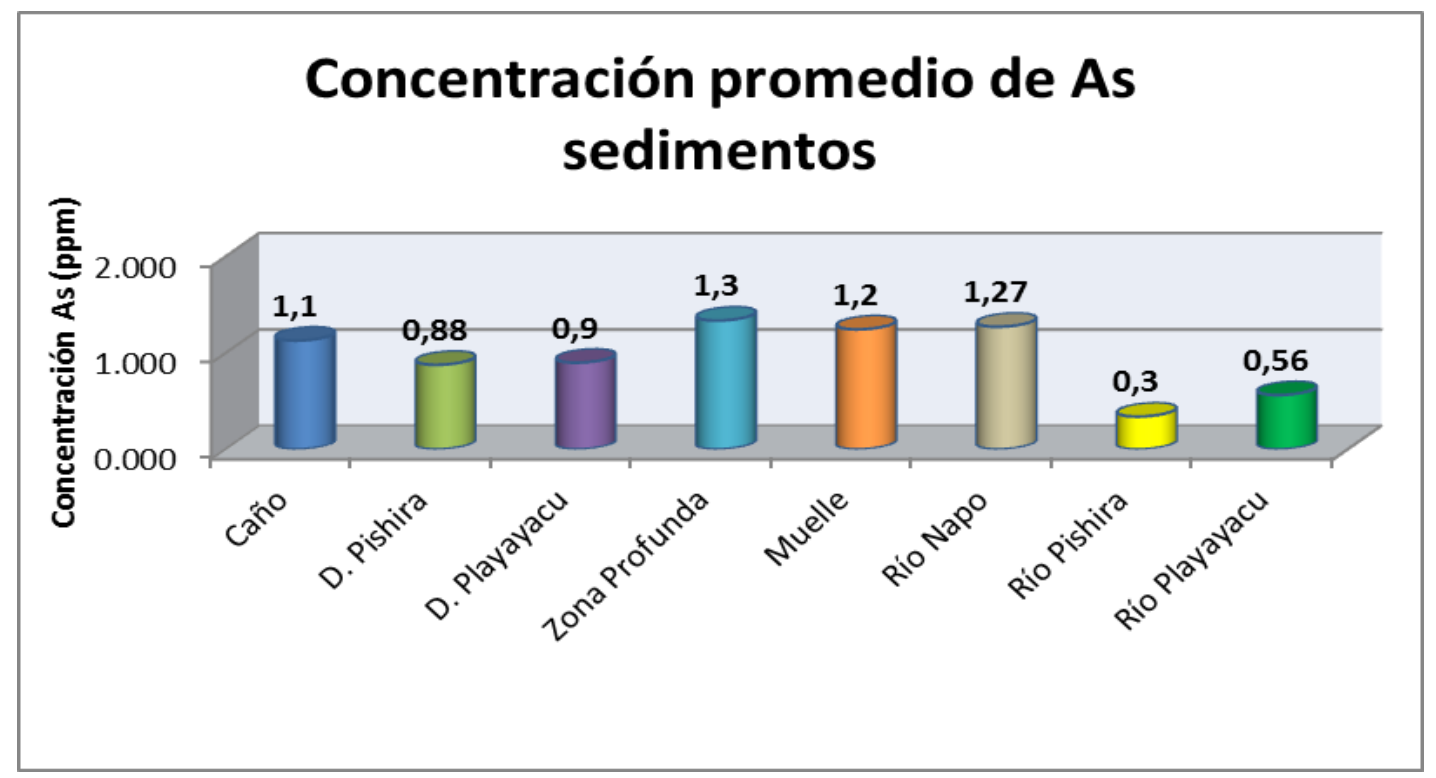

Figura 4. Concentración promedio de Arsénico en Sedimentos de la RBL (Elaborado por Carrillo D, 2016)

En cuanto a las concentraciones promedio de As en sedimentos, el punto Zona Profunda registra el valor más alto con $1,300 \mathrm{mg} / \mathrm{kg}$, seguido del río Napo con $1,270 \mathrm{mg} / \mathrm{kg}$ y con el menor valor el punto río Pishira con $0,300 \mathrm{mg} / \mathrm{kg}$, como se puede apreciar en la Figura 4.

El valor obtenido de fondo geoquímico fue de $0,220 \mathrm{mg} / \mathrm{kg}$, al realizar la comparación con las medianas de los puntos, se estableció que sobrepasan al valor del fondo geoquímico, como se visualiza en la Figura 5, donde también se realiza un paralelismo con las precipitaciones ocurridas en los mismos meses de realizado el muestreo. 


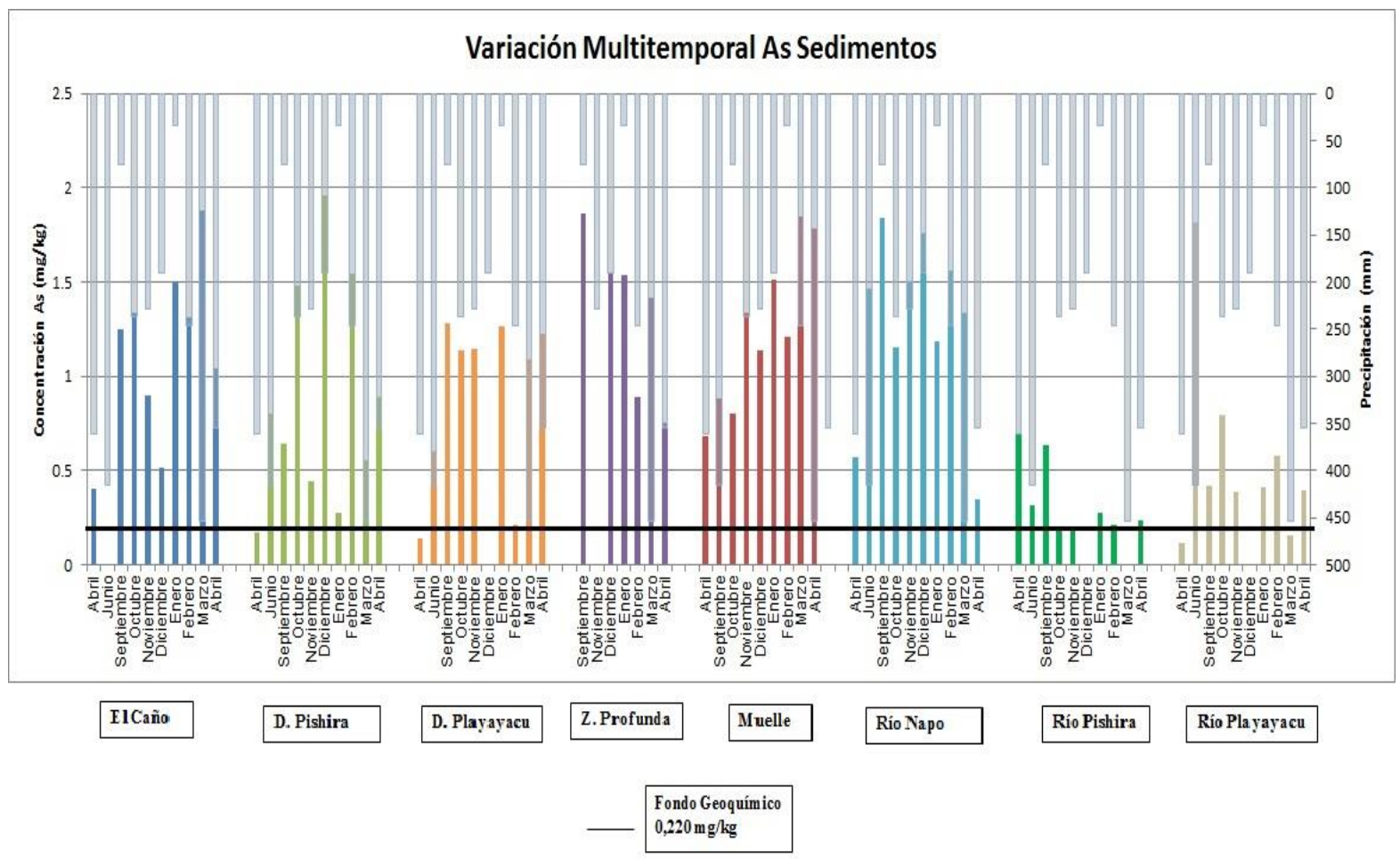

Figura 5. Variación multitemporal y multizonal del As en los sedimentos de la Reserva Biológica de Limoncocha (Elaborado por Carrillo D, 2016)

De lo analizado, no existe correlación directa entre las precipitaciones lluviosas en la zona, versus la concentración de As en los sedimentos, pero si se pudo apreciar variación multitemporal y multizonal en dicha concentración.

Los datos obtenidos de concentración de As en suelos de la RBL manifestaron un valor mínimo $0,038 \mathrm{mg} / \mathrm{kg}$ en el punto Instituto y como valor máximo $1,435 \mathrm{mg} / \mathrm{kg}$ en el punto Pozo Laguna, observándose dos valores atípicos para el punto Sendero "El Caimán"; mientras que temporalmente, existe un valor máximo en diciembre y mínimo en abril de 2015, al igual que en sedimentos, teniendo dos valores atípicos en los meses de junio y febrero. De los datos de concentración de abril del 2015 a abril 2016, ocho valores fueron no detectables por el método utilizado. En el mes de Diciembre 2015, por problemas climatológicos, fue imposible acceder a los puntos pozo Antiguo, pozo Laguna y pozo Jivino B para la recolección de muestra respectiva. Finalmente en los dos últimos meses de muestreo marzo y abril de 2016 en el punto pozo Jivino B no se pudo recolectar la muestra pues el lugar por el cual se accedía fue cerrado por PetroAmazonas. 


\section{Concentración promedio de As suelos}

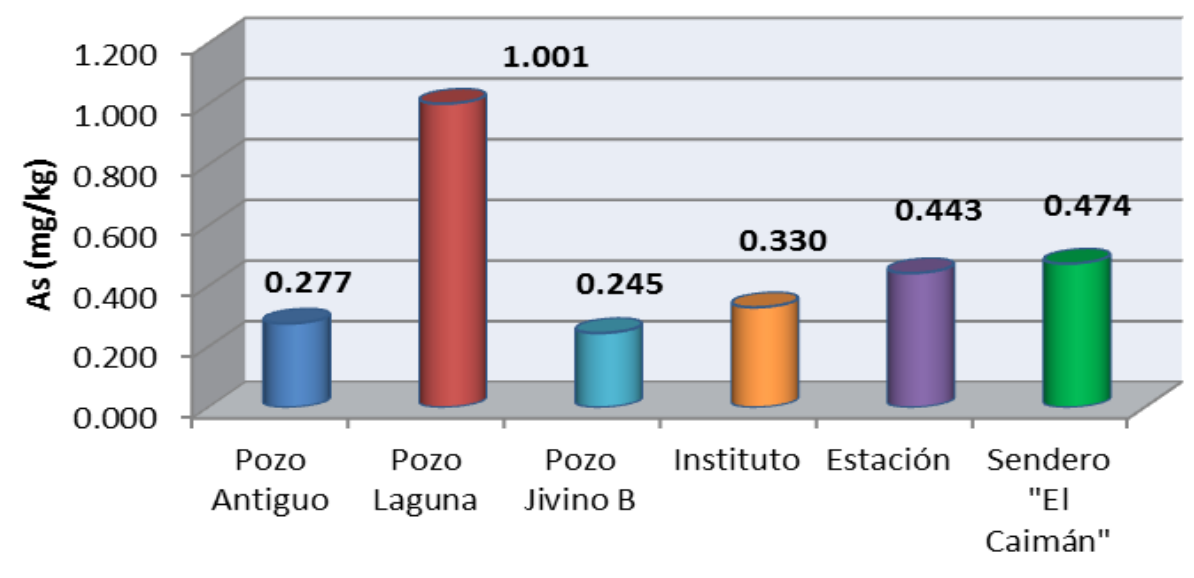

Figura 6. Concentración promedio de As en suelos de la RBL, (Elaborado por Carrillo D, 2016)

En la Figura 6, fueron representadas las concentraciones promedio de As enlos suelos muestreados de la RBL, así como el valor de fondo geoquímico tomado en el sendero El Caimán y cuyo valor es de $0.474 \mathrm{mg} / \mathrm{kg}$.

La Figura 7, muestra las variaciones multitemporales y multizonales de la concentración de As en los suelos de la RBL, encontrándose en el Pozo Laguna los valores más elevados en los meses de muestreo y análisis.

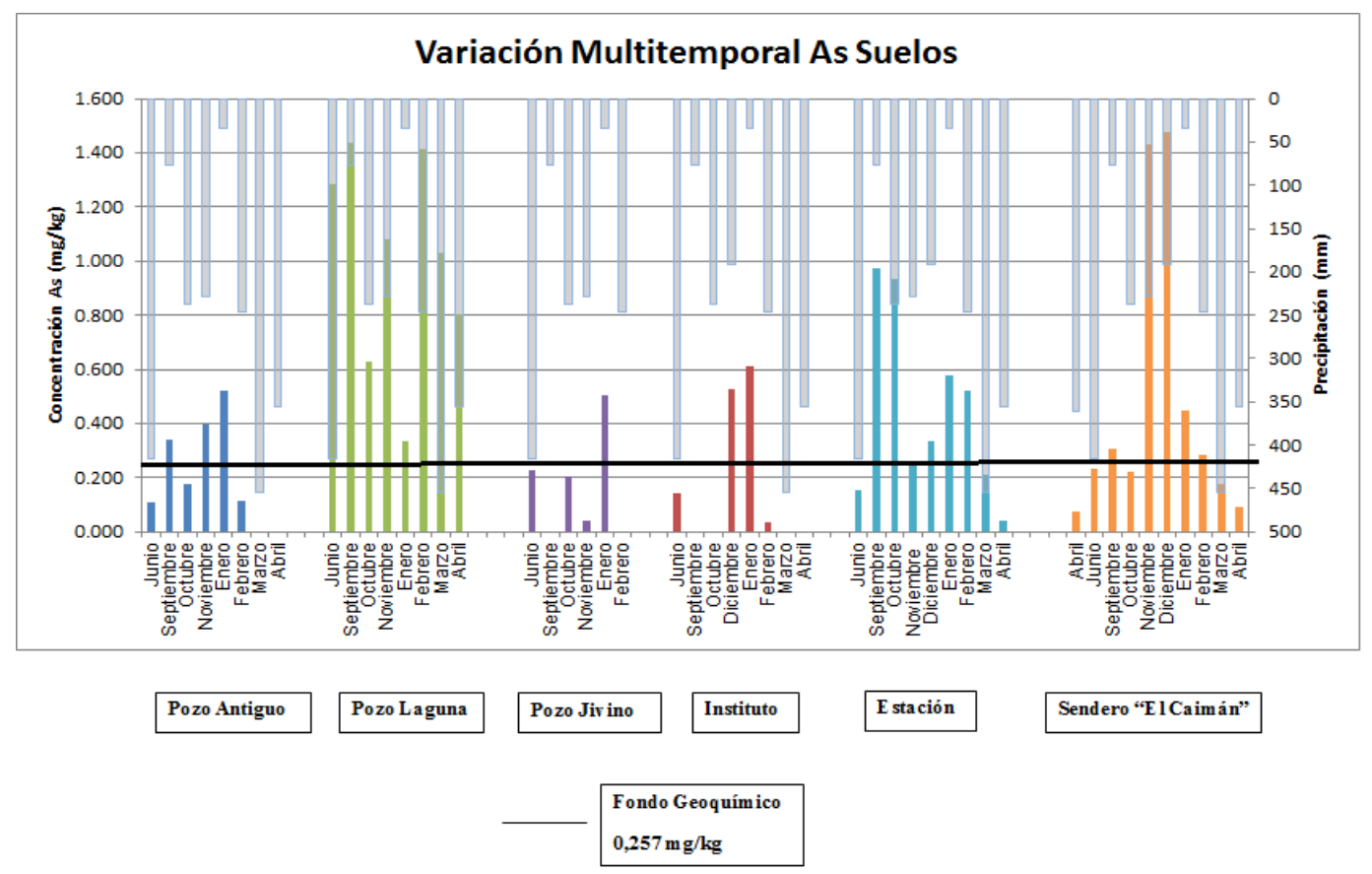

Figura 7. Variación multitemporal y multizonal del As en los suelos de la Reserva Biológica de Limoncocha. (Elaborado por Carrillo D, 2016) 


\section{Conclusiones y recomendaciones}

En las muestras de aguas no se detectó arsénico, pues sus concentraciones se encontraron por debajo del límite de detección teórico del equipo utilizado que es de $1 \mathrm{ppb}$, con estos valores inferiores al límite de detección, se puede concluir que las aguas naturales de la Reserva Biológica de Limoncocha, cumplen con la Normativa Ecuatoriana, Acuerdo Ministerial 097 que establece como límite máximo permisible de Arsénico para la Preservación de Flora y Fauna en aguas dulces, un valor de $0.05 \mathrm{mg} / \mathrm{L}$.

Las aguas de la Laguna están en contacto con los sedimentos y suelos de la RBL, en todos los cuales se detectó presencia de Arsénico, sin embargo el factor agua no presenta valores significativos de este elemento, por lo que se puede concluir que el As presente suelos y sedimento de la reserva, debe estar en una forma química que dificulta su solubilidad del mismo, y por lo tanto los coeficientes de transferencia de masa sedimento-agua deben ser bajos, recomendándose realizar ensayos de lixiviación con tiempos de contacto superiores a 24 horas para la determinación de estos coeficientes, estudio que se recomienda realizar a la brevedad posible.

Los suelos de la reserva Biológica de Limoncocha presentan concentraciones promedio diferentes de As, de acuerdo al punto de muestreo. Sin embargo, ninguno de estos valores sobrepasa la normativa Ecuatoriana, 097-A (2015); Anexo 2. Tabla 1: Criterios de calidad del suelo, que establece como límite máximo permisible de Arsénico para suelos un valor de 12 $\mathrm{mg} / \mathrm{kg}$. A pesar de ello, la misma norma también establece que los valores expuestos son referenciales y que siempre se deberá atender a los valores de fondo de cada sector.

El punto considerado fondo para suelos, por ser un punto no intervenido alejado de actividad antrópica, fue el Sendero El Caiman que presenta un valor promedio de As de 0.474 $\mathrm{mg} / \mathrm{kg}$ verificándose que existen valores de concentración de As, en todos los puntos exceptuando el punto pozo Jivino B, cuyas medianas sobrepasan el valor de la mediana del punto blanco, siendo el punto Pozo Laguna el de mayor valor con 1,055 mg/kg; por lo que para posteriores investigaciones se recomienda realizar la evaluación utilizando de referencia el valor de la mediana del punto Sendero "El Caimán. Por lo que se puede concluir que han incrementado los niveles de As en suelos de la RBL, pudiendo ser ocasionados por condiciones naturales, actividad antropogénica o industrial propia de la zona, por lo que el proceso investigativo debe continuar.

La legislación ecuatoriana no registra valores máximos permisibles para ningún parámetro en sedimentos, razón por la cual no se ha realizado a comparación de los valores de As con normativa y se ha optado por determinar el valor de fondo geoquímico que es de 0.220 $\mathrm{mg} / \mathrm{kg}$, habiéndose obtenido un valor promedio de As para los sedimentos de la reserva de 0.956 $\mathrm{mg} / \mathrm{kg}$. El estudio realizado durante un año, demuestra variación temporal y espacial de las concentraciones de As en los sedimentos de la Reserva Biológica de Limoncocha, al comprar las medianas de los puntos muestreados con el fondo geoquímico Figura 8., se determina que se ha producido un incrementado del valor de As en los sedimentos de la RBL, pudiendo deberse a causas tanto naturales como antrópicas, las cuales no pueden ser determinadas en este momento del estudio. 


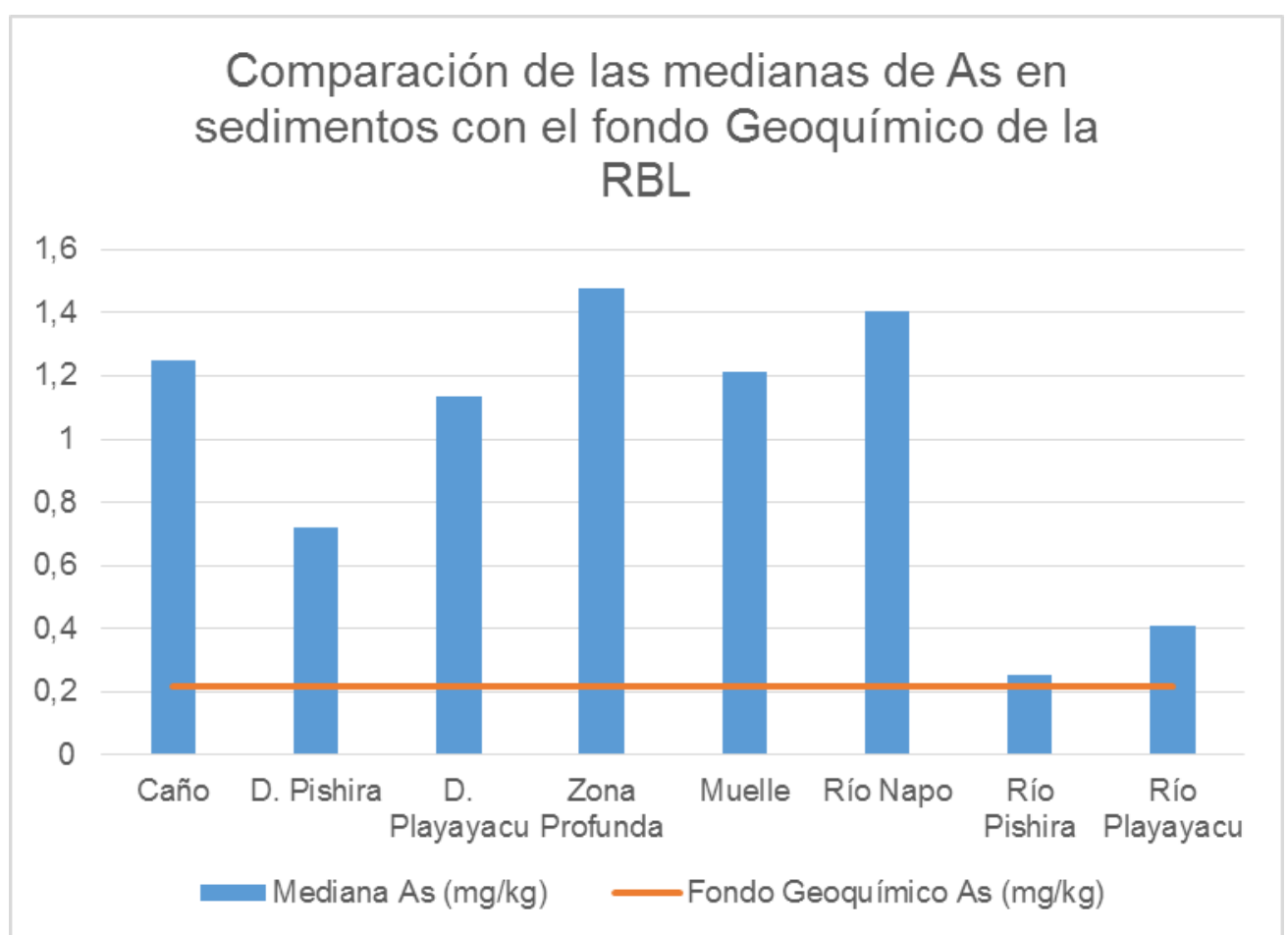

Figura 8. Comparación de las medianas de As en sedimentos con el Fondo Geoquímico de la RBL. (Elaborado por Coral K, 2019)

Para establecer la significancia ambiental de las concentraciones de As en los sedimentos de la Reserva, se buscó normativa a nivel mundial que establezca valores máximos permitidos de este elemento, se optó por usar la normativa canadiense que establece un valor de As para sedimentos de $5.9 \mathrm{mg} / \mathrm{kg}$, al comparar el promedio de los valores de arsénico en los sedimentos de la RBL con este valor, se obtuvo un valor menor a 1 (uno) con lo que, y siempre referido al valor de la norma Canadiense (ISQG. 2001), la concentración de As en los sedimentos de la RBL carece de significancia ambiental.

Si bien, este estudio ha permitido establecer valores referenciales de As para aguas, suelos y sedimentos de la RBL, que pueden ser considerados como línea base, esta debe ser cumplimentada en otros metales bajo la misma metodología establecida. La línea base es una herramienta que permitirá ubicar la condición fisicoquímica de la Reserva en términos de espacio, tiempo y afectación, para establecer su correcta gestión.

\section{Bibliografía}

Alarcón MT, Leal L, Martín I, Miranda S, Benavides A, 2013. “Arsénico en agua”, Centro de investigación en materiales avanzados, Chihuahua, Pg. 10.

Agencia para Sustancias Tóxicas y el Registro de Enfermedades. (ATSDR). 2014. "Reseña Toxicológica del Arsénico (versión actualizada) (en inglés)". Atlanta, GA: Departamento de Salud y Servicios Humanos de EE. UU. Servicio de Salud Pública. 
APHA AWWA WEF, Standard Methods. 2005. "Standard Methods: 3030 E: Nitric Acid Digestion of Metals". Standard Methods for the Examination of Water and Wastewater. 21st Edition.

Armas, M.F \& Lasso, S. 2011. “Plan de Manejo de la Reserva Biológica Limoncocha”. Ministerio del Ambiente. Pg. 104, 109.

Bravo, Elizabeth. 2007. "Los Impactos De La Explotación Petrolera En Ecosistemas Tropicales Y La Biodiversidad”. Acción Ecológica. Pg. 11, 18, 19, 20.

Cordovez, Beatriz. 1998. "Caracterización y Evaluación de los suelos y sedimentos del sector y de la Laguna de Limoncocha”. Trabajo de Fin de Carrera previo a la obtención del Título de Ingeniero Ambiental. UISEK. Quito, Ecuador.

EPA. 1996. "Method 3050B: Acid Digestion of Sediments, Sludges, and Soils," Revision 2.

Hernández-Crespo. C, Martín. M. 2015. "Determination of background levels and pollution assessment for seven metals $(\mathrm{Cd}, \mathrm{Cu}, \mathrm{Ni}, \mathrm{Pb}, \mathrm{Zn}, \mathrm{Fe}, \mathrm{Mn})$ in sediments of a Mediterranean coastal lagoon". Instituto de Ingeniería del Agua y Medio Ambiente, Universidad Politécnica de Valencia.

INAMHI. (2016). "Datos de Precipitación de la Estación Nuevo Rocafuerte”. Instituto Nacional de Meteorología e Hidrología. Quito, Ecuador.

ISQG. 2001. "Canadian Sediment Quality Guidelines for the Protection os aquatic life: Summary tables". Canadian Council Of Ministers of the Environment. Updated in: Canadian environmental Quality Guidelines, 1999, Canadian Council of Ministers of the Environment, Winnipeg.

Madera, Roberto. 2011. "Monitoreo De Caimanes (Alligatorinae): Aplicación En Estudios Poblacionales En La Laguna De Limoncocha, Provincia De Sucumbíos”. Trabajo de Fin de Carrera previo a la obtención del Título de Ingeniero Ambiental. UISEK. Pg. 21-24.

Ministerio del Ambiente. 2015. "Acuerdo Ministerial No. 097-A". Anexos del Texto Unificado de Legislación Secundaria del Ministerio del Ambiente. Edición Especial № 387 Registro Oficial. Pg. 14, 35.

Ministerio del Ambiente. 2015. "Reserva Biológica Limoncocha”. Sistema Nacional de Áreas Protegidas del Ecuador.

Neira, Francisco; Gómez, Santiago \& Pérez, Gloria. 2006. "Sostenibilidad de los usos de subsistencia de la biodiversidad en un área protegida de la Amazonia ecuatoriana: un análisis biofísico". Ecuador Debate. Debate Agrario. Pg. 156, 157 y 158.

Nordberg, Gunnar. (2014). "Metales: Propiedades Químicas y Toxicidad". Enciclopedia de Seguridad y Salud en el Trabajo. Parte IX. Productos químicos. Capítulo 63. Cuarta Edición. Pg. 2569- 2614.

Reyes, M. et al. (S.A). "Metales Pesados: Importancia Y Análisis”. Centro Interdisciplinario de Investigación para el Desarrollo Integral Regional, Unidad Durango, Instituto Politécnico Nacional. México. 